\title{
Actividad respiratoria de microorganismos en un suelo patagónico enmendado con lodos salmonícolas ${ }^{\#}$
}

\author{
Evolution of microbial respiratory activity in a Patagonian \\ soil amended with salmon-farming sludge

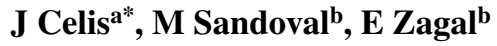 \\ aDepartamento de Ciencias Pecuarias, Facultad de Ciencias Veterinarias, Universidad de Concepción, Concepción, Chile. \\ bDepartamento de Suelos y Recursos Naturales, Facultad de Agronomía, Universidad de Concepción, Concepción, Chile.
}

\section{SUMMARY}

\begin{abstract}
Pollution of aquatic fresh water and soil is of great concern in the salmon farming industry. Water is the most important vehicle for the transmission of organic polluting agents, and for that reason the sediments (sludge) near to salmon facilities could become enriched reservoirs of pathogens and chemicals. A solution to this problem could be to incorporate the sludge into the soil under sustainable procedures. In order to investigate the addition of salmon sludge to soils, sediment samples from a pisciculture and a lake were applied to a degraded in the soil southern Chilean Patagonia. Microbial biological activity was evaluated under different salmon sludge addition rates: $25,50,75,100$ y $150 \mathrm{t} \mathrm{ha}^{-1}$ by using respirometry tests $\left(\mathrm{C}-\mathrm{CO}_{2}\right)$ in samples under closed incubation in laboratory. The results showed that microorganisms increased their respiratory activity along with time and increased sludge concentrations, indicating the amount of sludge that is possible to incorporate into a soil without causing adverse environmental effects.
\end{abstract}

Palabras clave: acuicultura, sedimentos, residuos orgánicos, eutroficación.

Key words: aquaculture, sediments, organic wastes, eutrophication.

\section{INTRODUCCIÓN}

Chile ha experimentado un explosivo aumento en la producción de salmónidos en los últimos años, pasando desde las 50.000 toneladas anuales que se producían en 1990 (Salazar y col 2005) a 397.000 toneladas el 2007, situándolo como el segundo mayor productor de salmónidos en el mundo ${ }^{1}$. Sin embargo, este extraordinario avance también ha generado importantes volúmenes de residuos orgánicos en los sistemas de crianza intensiva de salmones (Teuber y col 2007). Los desechos sólidos corresponden principalmente a alimento no consumido y a fecas de los peces que caen directamente al fondo del estanque (en el caso de la piscicultura) o al fondo del lago (caso de la salmonicultura lacustre). La salmonicultura chilena se concentra actualmente en el sur del país, principalmente en la X Región, y su crecimiento se está expandiendo rápidamente hacia la XI Región, donde se concentra gran parte de los suelos patagónicos. La Patagonia chilena comprende $108.000 \mathrm{~km}^{2}$, representando el $24 \%$ del territorio

Aceptado: 17.06.2009.

\# Financiado por Dirección de Investigación, U. de Concepción, Proyecto DIUC 208. 153.018-1.0

* Casilla 537, Chillán, Chile; jcelis@ udec.cl

1 Infante R. 2008. Industria del Salmón en Chile. http://200.9.100.74/ Rodrigo\%20Infante.pdf de Chile continental (Celis y col 2006). Esta región ha sido fuertemente intervenida por el hombre, dando lugar a una enorme cantidad de suelos degradados. Esto implica un gran potencial para usar estos suelos patagónicos, carentes de fertilidad, como reservorios para el reciclaje de lodos salmonícolas.

Para que estos lodos se puedan aplicar exitosamente en suelos como enmiendas orgánicas debe existir un alto grado de descomposición de la materia orgánica hasta llegar a su estabilización biológica, tarea que es desempeñada por una variedad de microorganismos, como microfauna (tales como los protozoarios), bacterias, hongos, actinomicetos y levaduras (Pinochet y col 2001). La estabilización biológica implica una inhibición del proceso de nitrificación. Los nitratos, resultantes de la nitrificación, son considerados uno de los contaminantes del agua subterránea y superficial (Sawyer y col 2000), y la reducción de estos por parte de los microorganismos en su respiración es un indicador de la estabilización de lodos.

La respiración es uno de los parámetros más frecuentemente usados para cuantificar actividad microbiana en el suelo (Zagal y col 2002), y que está directamente relacionada con la descomposición de residuos orgánicos provenientes de distintas fuentes (Sylvia y col 1998). La actividad metabólica que realizan los microorganismos en cualquier proceso de descomposición puede ser cuantificada por medición de la producción de $\mathrm{CO}_{2}$ o por el consumo de $\mathrm{O}_{2}$ (Alef 1995). El presente estudio tuvo como 
objetivo determinar la evolución del $\mathrm{CO}_{2}$ producido por los microorganismos en un suelo patagónico enmendado con distintas cantidades de lodos de piscicultura y de salmonicultura lacustre y su relación con la estabilidad de la materia orgánica.

\section{MATERIAL Y MÉTODOS}

\section{TRABAJO DE CAMPO}

Las muestras de lodos residuales correspondieron a dos sistemas de producción de una industria salmonícola de la Región de Los Lagos, Chile: (i) sedimentos extraídos desde estanques de acumulación de una piscicultura en tierra y, (ii) sedimentos lacustres tomados a $20 \mathrm{~m}$ de profundidad mediante draga bajo balsas-jaula. Las muestras del suelo patagónico fueron obtenidas a $20 \mathrm{~cm}$ de profundidad, provenientes del sector Coyhaique Alto $\left(45^{\circ} 46^{\prime} \mathrm{S}\right.$ y $\left.72^{\circ} 07^{\prime} \mathrm{O}\right)$, perteneciente a la Región Aysén del General Carlos Ibáñez del Campo. Corresponde a una estepa fría, ubicada en la vertiente oriental de la Cordillera de los Andes, hacia el límite con Argentina, donde el tipo de vegetación predominante es el coirón (Celis y col 2006).

\section{TRABAJO DE LABORATORIO}

En los laboratorios del Departamento de Suelos de la Universidad de Concepción (acreditado por la Comisión de Normalización y Acreditación de la Sociedad Chilena de la Ciencia del Suelo, desde el año 2000) se determinaron las características fisicoquímicas del suelo y lodos muestreados: densidad aparente (Da), pH, materia orgánica (MO), nitrógeno disponible $(\mathrm{N})$, fósforo $(\mathrm{P})$, potasio disponible $(\mathrm{K})$, capacidad de intercambio catiónico (CICE). Los metales pesados de los lodos salmonícolas fueron determinados en el Laboratorio de Química Ambiental del Centro EULA de la Universidad de Concepción (acreditado por el INN de acuerdo a la Norma Chilena NCh-ISO 17025. Of2001, desde el año 2003).

Las muestras de lodos y suelos fueron mantenidas a temperaturas inferiores a $10{ }^{\circ} \mathrm{C}$ hasta su procesamiento en el laboratorio. Luego fueron secadas a temperatura ambiente ( $15 \%$ humedad, base peso seco) y tamizadas con malla $2 \mathrm{~mm}$. Enseguida, se prepararon distintas mezclas lodo/suelo, a partir de varias partidas de $1 \mathrm{~kg}$ de suelo, a las cuales se les adicionaron lodos a distintas dosis: 25, 50, 75, 100 y $150 \mathrm{t} \mathrm{ha}^{-1}$ (base peso seco). Se usó un tratamiento control consistente en suelo sin lodo. Estas dosis definieron los tratamientos: T1, T2, T3, T4 у T5 (donde $\mathrm{T} 1$ correspondió a una adición de lodo al suelo a razón de $25 \mathrm{t} \mathrm{ha}^{-1}$, y así sucesivamente). Estas mezclas lodo/suelo se homogeneizaron y se envasaron en bolsas plásticas, para luego someterlas a preincubación por 10 días, en cámara con temperatura $\left(25 \pm 2{ }^{\circ} \mathrm{C}\right)$ y humedad (60-70\%) controladas.
Enseguida, las mezclas preparadas se utilizaron para la determinación de la respiración de suelo como medida de la actividad microbiana mediante incubación cerrada en laboratorio, siguiendo el procedimiento descrito por Zagal y col (2002). Para el proceso de incubación se utilizaron muestras de $25 \mathrm{~g}$ de la mezcla lodo/suelo, las que fueron condicionadas previamente a capacidad de campo, y luego se colocaron dentro de frascos de vidrio de $1 \mathrm{~L}$ de capacidad (del tipo Mason jars). Se utilizó un total de 44 frascos, considerando los tratamientos (5), el control, el tipo de lodos (2) y las réplicas (4). En cada uno de ellos se ubicó un tubo de ensayo con $15 \mathrm{~mL}$ de solución $1 \mathrm{M}$ de $\mathrm{NaOH}$ para absorber el $\mathrm{CO}_{2}$ desprendido por los microorganismos, y otro con agua destilada para mantener la atmósfera húmeda. Cada frasco se selló herméticamente con parafilm. Luego los frascos fueron llevados a cámara de incubación y mantenidos a $22{ }^{\circ} \mathrm{C}$ por 60 días. Se realizaron mediciones de la cantidad de $\mathrm{NaOH}$ remanente (no neutralizado) a través del tiempo de incubación, mediante titulación con $0,1 \mathrm{M}$ de $\mathrm{HCl}$, reemplazando la respectiva trampa de $\mathrm{CO}_{2}$ por una nueva en cada ocasión. Para la titulación de la solución se extrajo (en duplicado) $1 \mathrm{~mL}$ de $\mathrm{NaOH}$, y se adicionaron $2 \mathrm{~mL}$ de $\mathrm{BaCl}_{2}$ para precipitar el $\mathrm{C}$ inorgánico como $\mathrm{BaCO}_{3}$ insoluble. Se adicionaron gotas de fenolftaleína como indicador ácido base y se tituló el $\mathrm{NaOH}$ no neutralizado directamente con $\mathrm{HCl}$ (Ianotti y col 1994). La cantidad de $\mathrm{CO}_{2}$ desprendido desde las mezclas incubadas fue calculada mediante la fórmula de Anderson (1982).

\section{ANÁLISIS DE LOS RESULTADOS}

Los resultados fueron expresados en $\mu \mathrm{g}$ C- $\mathrm{CO}_{2} \mathrm{~g}^{-1}$ de suelo y como mg C- $\mathrm{CO}_{2} \mathrm{~g}^{-1} \mathrm{~d}^{-1}$ de materia orgánica (del suelo y lodo), en base peso seco (horno a $105^{\circ} \mathrm{C}$ ). Los datos se procesaron mediante análisis de varianza ANDEVA, y las comparaciones de medias fueron realizadas de acuerdo al test de Tukey. Se usó el programa SAS versión 8.1 para Windows (SAS Institute Inc, Cary, NC, USA). En todos los casos el nivel de significancia utilizado fue de $\mathrm{P}<0,05$.

\section{RESULTADOS Y DISCUSIÓN}

Los análisis fisicoquímicos (cuadro 1) revelaron que el suelo patagónico presenta un bajo contenido de MO, $\mathrm{N}$ y $\mathrm{P}$, típico de los suelos degradados, mientras que los lodos de salmonicultura tienen altas cantidades de MO, N y $\mathrm{P}$, siendo estos valores más altos en el caso del lodo de piscicultura. El pH, importante, pues puede llegar a cambiar las poblaciones microbianas (Coria y col 2007), es casi neutral, lo cual es bueno ya que condiciones ácidas reducen la tasa de descomposición de MO (Bernal y col 1998).

En la figura 1 se muestra la evolución del C- $\mathrm{CO}_{2}$ en el suelo patagónico enmendado con distintas dosis de lodos salmonícolas. En general, se observó un aumento no lineal 
Cuadro 1. Composición física y química del suelo patagónico y lodos salmonícolas. salmon sludge.

Physical and chemical composition of Patagonian soil and

\begin{tabular}{lrcc}
\hline & Suelo & $\begin{array}{c}\text { Lodo } \\
\text { piscicultura }\end{array}$ & Lodo lago \\
\hline $\mathrm{Ma}\left(\mathrm{kg} \mathrm{m}^{-3}\right)$ & 1,0 & 0,9 & 0,8 \\
$\mathrm{pH}(\mathrm{al} \mathrm{agua})$ & 6,8 & 7,0 & 6,7 \\
$\mathrm{MO}(\%)$ & 2,9 & 20,7 & 18,3 \\
$\mathrm{~N}\left(\mathrm{mg} \mathrm{kg}^{-1}\right)$ & 12,1 & $1.695,3$ & 746,0 \\
$\mathrm{P}\left(\mathrm{mg} \mathrm{kg}^{-1}\right)$ & 3,2 & 480,0 & 19,9 \\
$\mathrm{~K}\left(\mathrm{mg} \mathrm{kg}^{-1}\right)$ & 414,3 & 30,0 & 120,8 \\
$\mathrm{CICE}\left(\mathrm{cmol} \mathrm{kg}^{-1}\right)$ & 8,9 & 1,7 & 19,3 \\
$\mathrm{C} / \mathrm{N}$ & 13,4 & 10,5 & 7,5 \\
\hline
\end{tabular}

en la actividad respiratoria de los microorganismos a través del tiempo, similar a otros estudios (Zagal y col 2002, Guerrero y col 2007). Excepto el control, en todas las curvas se destaca una primera fase (de pendiente fuerte, hasta los 30 días) en donde la respiración es más rápida y una segunda fase (de pendiente suave, entre los 30 a los 60 días) en donde el incremento es más lento. Hubo una mayor actividad respiratoria a medida que aumenta la dosis de lodo adicionada al suelo, siendo todos los tratamientos (T1 a T5) mayores que el control. En el caso de las enmiendas con lodos de piscicultura, a los 15 días de incubación los tratamientos mostraron entre 6 y 32 veces mayor actividad que el control, mientras que a los 60 días estas diferencias fueron menores (entre 5 y 24). Los mayores valores de respiración sugieren una mayor disponibilidad de $\mathrm{C}$ y energía para los microorganismos heterótrofos del suelo, consecuencia de un suministro abundante de materia orgánica y nitrógeno, lo cual favoreció la actividad de los microorganismos (Zagal y col 2002). Es por ello que, siguiendo el mismo patrón, los tratamientos con lodo lacustre mostraron menores valores que las enmiendas con lodos de piscicultura, lodo este último con contenidos de $\mathrm{N}$ y $\mathrm{P}$ más bajos que el lodo de lago (cuadro 1).

Los resultados de respirometría como consecuencia de las enmiendas realizadas en suelos agrícolas sugieren un efecto positivo para el crecimiento y actividad microbiana, situación que ha sido reportada por numerosos autores (Zagal y col 2002, Ros y col 2003, Varnero y col 2004, Teuber y col 2007). Según Esparza y col (2004), suelos con bajo contenido de MO, como es el caso del suelo patagónico estudiado (cuadro 1), muestran una mayor respuesta a la adición de lodos. La incorporación de residuos orgánicos en condiciones de humedad y aireación favorece la presencia de microorganismos que inicialmente descomponen rápidamente el material y que posteriormente disminuyen la tasa de descomposición (Barrena y col 2006).

A medida que el contenido de materia orgánica en el sustrato fue disminuyendo, la tasa de respiración de microorganismos en el lodo de piscicultura y en el lodo lacustre (figura 2) disminuyó durante el proceso de incubación,
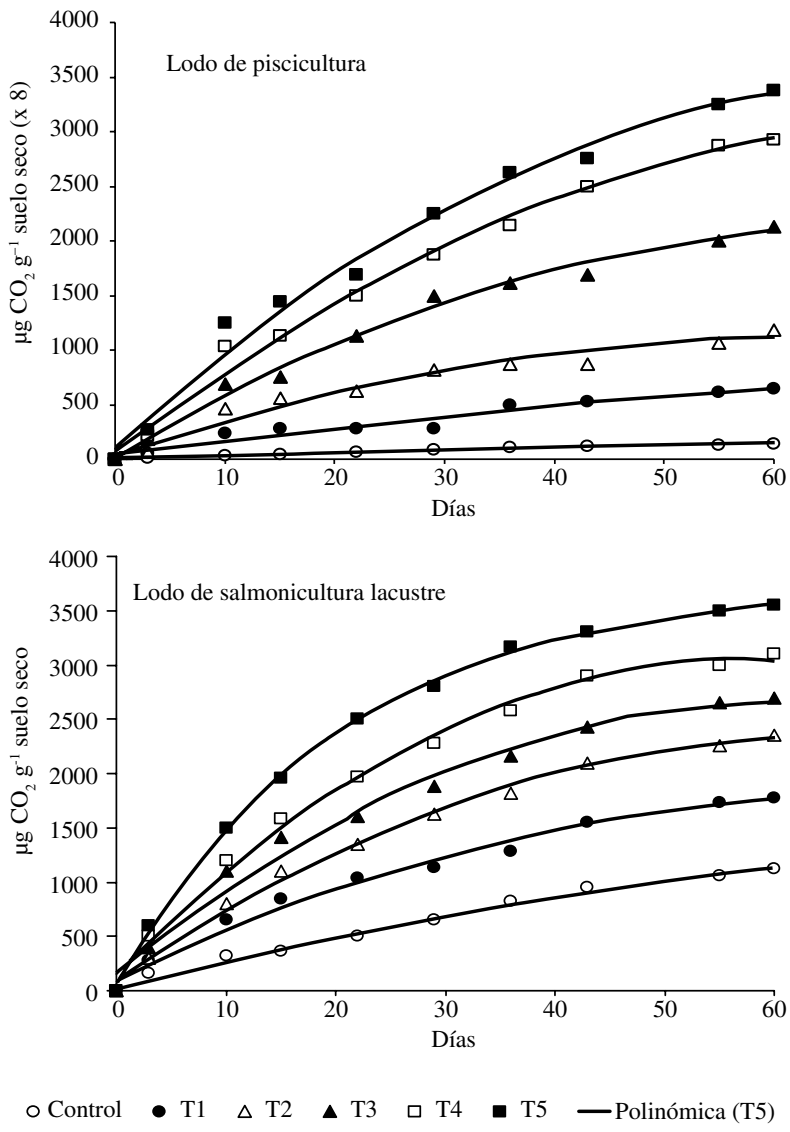

Figura 1. Evolución acumulada de $\mathrm{C}-\mathrm{CO}_{2}$ medida (símbolos) y ajustada (líneas continuas) durante la incubación in vitro de muestras superficiales $(0-20 \mathrm{~cm})$ no disturbadas de un suelo patagónico bajo distintas enmiendas con lodos salmonícolas (T1 a T5).

Measured (symbols) and adjusted (solid lines) accumulated $\mathrm{C}-\mathrm{CO}_{2}$ evolution during the in vitro incubation of a Patagonian undisturbed superficial $(0-20 \mathrm{~cm})$ soil samples under different amendments with salmon sludge (T1 to T5).

para todos los tratamientos. Similares resultados fueron observados por Wang y col (2004), quienes trabajaron con residuos orgánicos de lechería y porcinos. En el caso de las incubaciones con lodo de piscicultura, se obtuvieron valores estables del orden de los $2 \mathrm{mg} \mathrm{CO}_{2} \mathrm{~g}^{-1} \mathrm{MO} \mathrm{d}^{-1}$, lo cual ocurrió a los 55 días. La estabilidad biológica de un residuo orgánico se establece cuando no supera $\operatorname{los} 8 \mathrm{mg}$ $\mathrm{C}-\mathrm{CO}_{2} \mathrm{~g}^{-1} \mathrm{MO} \mathrm{d}^{-1}$, que es cuando ya los microorganismos han agotado el stock de MO fácilmente descomponible (Varnero y col 2004). Proyectando dicho valor en la figura 2 correspondiente al lodo de piscicultura, resulta que habría madurez biológica a los 10, 15, 20, 38 y 45 días cuando este tipo de lodo es aplicado a dosis de 25, 50, 75, 100 y $150 \mathrm{t} \mathrm{ha}^{-1}$, respectivamente. A medida que aumenta la carga de lodo aplicada al suelo también aumenta el tiempo que demora en alcanzar la madurez biológica del mismo. Esto significa que si se aplica lodo a razón de $150 \mathrm{t} \mathrm{ha}^{-1}$, 
demora 35 días más en estabilizarse que si este lodo se aplicara a una dosis de $25 \mathrm{t} \mathrm{ha}^{-1}$. Comparativamente, en el caso del lodo lacustre se obtuvieron valores estables del orden de los $2 \mathrm{mg} \mathrm{CO}_{2} \mathrm{~g}^{-1} \mathrm{MO} \mathrm{d}^{-1}$ a los 35 días (figura 2). La estabilidad biológica del lodo de lago se alcanzó a los $7,10,12,15$ y 20 días cuando se aplicó en dosis de 25, $50,75,100$ y $150 \mathrm{t} \mathrm{ha}^{-1}$, respectivamente, es decir, en un menor tiempo que el lodo de piscicultura. Esta más rápida estabilización puede deberse al hecho de que este tipo de lodo se obtuvo debajo de las jaulas en el fondo del lago, lugar donde estuvo expuesto a procesos previos de descomposición anaerobia que permitieron "premadurar" el lodo (Teuber y col 2005). Es importante alcanzar la estabilización biológica pues implica una menor producción de nitratos, considerados uno de los contaminantes del agua subterránea y superficial (Sawyer y col 2000).

La incorporación de materiales orgánicos, tales como lodos residuales, puede provocar un aumento en la biomasa microbiana del suelo al incorporar una nueva carga microbiana al sistema o al estimular el crecimiento de la microbiota autóctona por la incorporación de nuevas fuentes de carbono (Ros y col 2006). Sin embargo, otros estudios revelan una disminución en los niveles
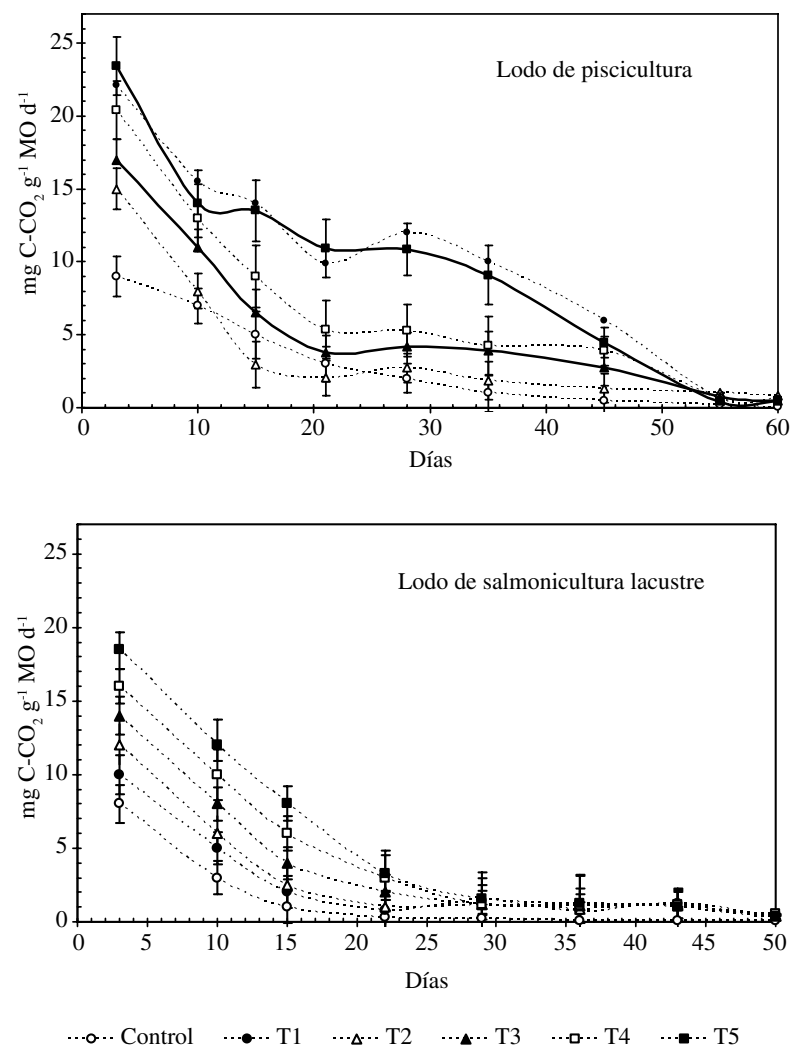

Figura 2. Cambios en la tasa de respiración de microorganismos durante el proceso de incubación de un suelo patagónico bajo distintas enmiendas con lodos salmonícolas (T1 a T5).

Changes in rate of $\mathrm{CO}_{2}$ respiration during incubation of a Patagonian soil under different amendments with salmon sludge (T1 to T5). de biomasa microbiana y una alteración en la diversidad y en la estructura de las comunidades del suelo debido a la presencia de metales pesados que a veces pueden contener los lodos, como también metales pesados introducidos debido a diferentes prácticas de manejo (Jonsen y col 2001, Crecchio y col 2004). Los análisis realizados a los lodos de salmonicultura utilizados en este estudio (cuadro 2) mostraron bajos niveles de metales pesados, lo cual revela que en este tipo de lodos no hubo impedimento para que los microorganismos se desarrollaran en perfectas condiciones.

Estos resultados, aunque de carácter exploratorio, demuestran que las aplicaciones de lodos salmonícolas, especialmente aquellos provenientes de piscicultura, estimularon la actividad microbiana en el suelo patagónico, incrementándose en directa relación con aumentos en las dosis enmendadas. Se observaron diferentes tasas de descomposición que estuvieron determinadas por la cantidad y el tipo de lodo aplicado al suelo. Así, mayores precauciones debieran tomarse cuando se trata de aplicar lodos provenientes de una piscicultura, ya que de acuerdo a las pruebas de respirometría demoran más tiempo en estabilizarse.

\section{RESUMEN}

La contaminación de los suelos y de los ecosistemas acuáticos de agua dulce es una preocupación constante para la industria de la salmonicultura. El agua es el vehículo más importante para la transmisión de contaminantes orgánicos, y por ello los sedimentos (lodos) próximos a las instalaciones de una piscicultura o salmonicultura pueden convertirse en reservorios de patógenos y químicos. Una solución es enmendar estos lodos en suelos de manera sustentable. Para investigar la adición de lodos residuales de salmonicultura en suelos, muestras de sedimentos obtenidos de piscicultura y de lago fueron aplicadas a un suelo patagónico degradado del sur de Chile. Se evaluó la actividad biológica de los microorganismos bajo diferentes dosis de lodos enmendados al suelo: 25, 50, 75, 100 y 150 $\mathrm{t} \mathrm{ha}^{-1}$. La determinación se realizó mediante pruebas de respirometría $\left(\mathrm{C}-\mathrm{CO}_{2}\right)$ en muestras sometidas a incubación cerrada en laboratorio por

Cuadro 2. Concentraciones de metales pesados $\left(\mathrm{mg} \mathrm{kg}^{-1}\right)$ en lodo de piscicultura y lodo de salmonicultura lacustre (lago).

Heavy metals concentrations $\left(\mathrm{mg} \mathrm{kg}^{-1}\right)$ pisciculture-salmon waste and lake-salmon waste.

\begin{tabular}{lccr}
\hline \multirow{2}{*}{ Metal pesado } & \multicolumn{2}{c}{ Lodo residual } & \\
\cline { 2 - 3 } & Piscicultura & Lago & \\
\hline Arsénico (As) & 0,73 & 0,94 & 40 \\
Cadmio (Cd) & 0,69 & 1,77 & 40 \\
Cromo (Cr) & 8,68 & 9,7 & 1.500 \\
Cobre (Cu) & 29,0 & 24,3 & 1.200 \\
Plomo (Pb) & 2,05 & 29,98 & 400 \\
Mercurio (Hg) & 0,024 & 0,15 & 20 \\
Níquel (Ni) & 24,5 & 7,4 & 420 \\
Selenio (Se) & 0,81 & 1,16 & 100 \\
Zinc (Zn) & 390,4 & 364,8 & 2.800 \\
\hline
\end{tabular}

1 Concentraciones límites en lodos aplicados a suelos (Castro y col 2007). 
60 días. Los resultados mostraron que los microorganismos aumentan su actividad respiratoria a medida que transcurre el tiempo y con mayores tasas de aplicación de lodo, indicando con ello la cantidad de lodo residual que es posible disponer o verter en un suelo sin provocar efectos ambientales adversos.

\section{REFERENCIAS}

Alef K. 1995. Soil respiration. In: Alef K, Nannipieri P (eds). Methods in applied soil microbiology and biochemistry. Academic Press Limited, San Diego, USA, Pp 214-218.

Anderson JP. 1982. Soil respiration. In: Page AL, Miller RH, Keeney DR (eds). Methods of soil analysis: Chemical and Microbiological properties. $2^{\text {nd }}$ ed. American Society of Agronomy Inc, Wisconsin, USA, Pp 831-871.

Barrena R, F Vázquez, A Sánchez. 2006. The use of respiration indices in the composting process: a review. Waste Manage Res 24, 37-47.

Bernal MP, MA Sánchez-Monedero, C Paredes, A Roig. 1998. Carbon mineralization from organic wastes at different composting stages during their incubation with soil. Agr Ecosyst Environ 69, 175-189.

Castro C, O Henríquez, R Freres. 2007. Posibilidades de aplicación de lodos o biosólidos a los suelos del sector norte de la Región Metropolitana de Santiago. Rev Geogr Norte Gd 37, 35-45.

Celis J, M Sandoval, E Zagal, M Briones. 2006. Efecto de la adición de biosólidos urbanos y de salmonicultura sobre la germinación de semillas de lechuga (Lactuca sativa L.) en suelo patagónico. $R C$ Suelo Nutr Veg 6, 13-25.

Coria ML, JP Fay, SB Cseha, MA Brizuela. 2007. Efecto de concentraciones elevadas de sales totales y sulfatos en agua de bebida sobre la degradabilidad ruminal in vitro de Thinopyrum ponticum. Arch Med Vet 39, 261-267.

Crecchio C, A Gelsomino, R Ambrosoli, JL Minati, P Ruggiero. 2004. Functional and molecular responses of soil microbial communities under differing soil management practices. Soil Biol Biochem 36, 1873-1883.

Esparza J, M Diez, F Gallardo. 2004. Aplicación de lodos de la industria de celulosa a suelos Andisoles chilenos. Simposio Residuos Orgánicos y su Uso en Sistemas Agroforestales. Universidad de La Frontera, Temuco, Chile, Pp 27-38.
Guerrero C, R Moral, I Gómez, R Zornoza, V Arcenegui. 2007. Microbial biomass and activity of an agricultural soil amended with the solid phase of pig slurries. Biores Technol 98, 3259-3264.

Ianotti D, T Pang, B Toth. 1994. A quantitative respirometric method for monitoring compost stability. Compost Sci Utiliz 1, 52-65.

Jonsen K, CS Jacobsen, V Torsvik. 2001. Pesticide effects on bacterial diversity in agricultural soils. Biol Fertil Soils 33, 443-453.

Pinochet D, P Artacho, P Azúa. 2001. Potencialidad como abono orgánico de los desechos sólidos subproductos del cultivo de especies salmonídeas. Agro Sur 29, 78-82.

Ros M, MT Hernández, C García. 2003. Soil microbial activity after restoration of a semiarid soil by organic amendments. Soil Biol Biochem 35, 463-469.

Ros M, JA Pascual, C García, MT Hernández, H Insam. 2006. Hydrolases activities, microbial biomass and bacterial community in a soil after long-term amendment with different compost. Soil Biol Biochem 38, 3443-3452.

Sawyer C, P McCarty, G Parkin. 2000. Chemistry for environmental engineering. McGraw-Hill Inc, New York, USA.

Sylvia D, J Fuhrmann, P Hartel, D Zuberer. 1998. Principles and applications of soil microbiology. Prentice Hall Inc., New Jersey, USA.

Teuber N, M Alfaro, F Salazar, C Bustos. 2005. Sea salmon sludge as fertilizer: effects on a volcanic soil and annual ryegrass yield and quality. Soil Use Manage 21, 432-434.

Teuber N, F Salazar, M Alfaro, A Valdebenito. 2007. Efecto de diferentes dosis de lodos de la crianza de salmones en el cultivo de papa y su efecto residual en ballica anual. Agric Téc (Chile) 67, 393-400.

Varnero MT, P Faúndez, C Santibáñez, P Alvarado. 2004. Evaluación de lodo fresco y compostado como materia prima para la elaboración de sustratos. En: Simposio Residuos Orgánicos y su Uso en Sistemas Agroforestales. Universidad de La Frontera, Temuco, Chile, Pp 477-481.

Wang P, CM Changa, ME Watson, WA Dick, Y Chen, HAJ Hoitink. 2004. Maturity indices for composed dairy and pig manures. Soil Biol Biochem 36, 767-776.

Zagal E, N Rodríguez, I Vidal, L Quezada. 2002. Actividad microbiana en un suelo de origen volcánico bajo distinto manejo agronómico. Agric Téc (Chile) 62, 297-309. 